\title{
Adaptation with constant and variable delay in auditory feedback
}

SAMUEL FILLENBAUM

UNIVERSITY OF NORTH CAROLINA

\begin{abstract}
The effects of an irregularly varying delay in auditory feedback (VDAF) were compared with those of a constant delay in feedback (DAF) with regard to: (a) impairment in level of performance, (b) adaptation in performance, and (c) possible differences in rate of adaptation. Performance in a reading task was compared using a DAF interval, of $.20 \mathrm{sec}$. and a VDAF interval varying irregularly from .10 to $.28 \mathrm{sec}$. No difference was found between conditions in amount of impairment in performance. There was a significant improvement upon continued reading in both conditions, and there was no difference between conditions in the rate of improvement.
\end{abstract}

\section{Problem}

There is much evidence indicating that delayed auditory feedback (DAF) may have various deleterious effects on speech (see Yates, 1963 for a review of the literature). In studies of DAF the common procedure has been to use some particular constant delay interval which is the same at both ears. The purpose of this experiment is assess the effects on speech (reading) of variable delay in auditory feedback (VDAF). This study seeks to explore the possible effects of one major parameter of the DAF situation which has not yet been investigated. It was hoped that the results might have some implications for the general interpretation of the effects of DAF. Thus if VDAF yielded results quite similar to those obtained with DAF, this might be considered as evidence against some versions of the mis-match hypothesis, which, in one form or other, constitutes the most common attempt to explain the effects of DAF in terms of asynchrony between different sources of feedback (see Yates, 1963)。Finally we were interested in the possibility of an analogy between the DAF situation and the work on adaptation to conditions of visual rearrangement. It has been shown that with displacement of the visual field, by use of deviating prisms, adaptation does occur if there is some orderly relation "between sensation and the sensory consequences of self produced motion" (Teuber, 1960), but that adaptation becomes impossible if the rearrangement is a variable one (with static prisms replaced by rotating prisms whose power is changed continuously). If the analogy holds, then perhaps one might expect adaptation under conditions of DAF but not under conditions of VDAF。

\section{Method}

Design. The design of the study is shown in Table 1. All Ss first read under normal conditions and then read under conditions of delayed feedback, the second reading serving as a warm-up task to acquaint them with the DAF situation. Then $\mathrm{S} s$ serving in Condition
I read six times more, the first and last of these readings being under conditions of VDAF, the other readings under conditions of DAF. Those Ss who served in Condition II al so read six more times, the first and last of these readings being under conditions of DAF, the other readings under conditions of VDAF.

Subjects and Materials. There were $42 \mathrm{Ss}$, with 21 serving in each of the two conditions. The $S$ s were recruited from the introductory courses in psychology. The material to be read consisted of 100 color names laid out in 10 rows of 10 names each. By permuting rows and columns we made up eight sheets, each containing the same items, but in different arrangement. The order of these eight sheets was separately shuffled for each $S$.

Apparatus and Procedure. A Bell and Howell delayed feedback recorder was employed. The delay interval could be pre-set, or could be manually changed while the recorder was operating by turning a knurled knob which governed the distance between the recording and play-back heads. Under conditions of DAF the delay interval was always pre-set at $.20 \mathrm{sec}$. Under conditions of VDAF the delay interval was varied in smooth but non-periodic fashion from $.10 \mathrm{sec}$. to $.28 \mathrm{sec}$. Thus the interval might be varied from .28 to $.20 \mathrm{sec}$. and then more slowly to $.25 \mathrm{sec}$, before being shifted down to $.15 \mathrm{sec}$. or .10 sec., etc. A pre-determined routine was followed by $E$ in irregularly varying the delay interval.

Upon arrival $\mathrm{S}$ was seated, and told he would be given a number of reading tasks. He was positioned in front of the microphone, and the microphone and earphones were adjusted. Each $S$ was told that he was to read aloud at his usual rate and in his normal voice. He then read the color names on the first sheet under normal conditions. After this he was told that he would now be given a number of further "passages" like the one he had just read and that "During this part of the experiment you will hear your own voice coming through the earphones in an unaccustomed way. Regardless of what you hear through the earphones try to continue speaking at your usual rate and in your normal voice. Let me repeat this for it is important: you are always to try to speak at your usual rate and in your normal voice." S now read the warm-up "passage," and then the other six "passages." There was a $1 \mathrm{~min}$. pause after each reading during which the relevant part of the instructions was repeated to $S$. On each occasion, except for the warm-up "passage," reading time was recorded to the nearest half second.

Data Analysis. To permit a levels by treatments analysis which takes account of variance attributable to differences in reading time under normal reading conditions, $S s$ in each of the two conditions were assigned to one of three levels in terms of reading time under normal conditions. These levels were (a) $60 \mathrm{sec}$. or less, (b) 60.5 . $74.5 \mathrm{sec} .,(c) 75 \mathrm{sec}$. or more. This resulted in seven Ss being assigned to each level in each condition. ${ }^{2}$ With original reading time thus used as a control variable, analyses were then carried out on reading times under different conditions of delay in feedback.

\section{Results and Diseussion}

Mean reading times by condition and trial are shown in Table 1. The analyses of variance yielded the following results: (a) $\mathrm{T}_{1}$ vs $\mathrm{T}_{2}$ (assessing initial differences between DAF and VDAF and any effects of shift from one to the other)-the only significant effect $(p<.01)$ was that attributable to levels (differences in reading time under normal conditions); (b) $\mathrm{T}_{1}$ vs $\mathrm{T}_{6}$ (assessing adaptation effects with intervening reading under different conditions) - the levels effect was again 
TABLE I

EXPERIMENTAL DESIGN AND MEAN READING TIMES IN SECONDS

\begin{tabular}{|c|c|c|c|c|c|c|c|}
\hline & & $T_{1}$ & $T_{2}$ & $T_{3}$ & $\mathrm{~T}_{4}$ & $\mathrm{~T}_{5}$ & $T_{6}$ \\
\hline ondition 1 & Normal: 69.5 & VDAF: 83.1 & DAF.20: 83.8 & DAF.20: 82.8 & DAF.20: 79.5 & DAF.20: 77.2 & VDAF: 75.6 \\
\hline Condition II & Normal: 67.0 & DAF.20: 85.3 & VDAF: 86.9 & VDAF: 86.8 & VDAF: 83.2 & VDAF: 79.2 & DAF. $20: 76.6$ \\
\hline
\end{tabular}

significant $(p<.01)$, as was that attributable to trials $(p<.01)$ indicating a significant decrease in reading time (adaptation) from $T_{1}$ to $T_{6}$, no other effect was significant; (c) trend analyses on $T_{2}, T_{3}, T_{4}, T_{5}$ (assessing adaptation effects and possible differences in adaptation between conditions)-the between group means effect was significant $(p<.01)$ this being due to the levels effect which was also significant $(p<.01)$, overall trend was significant $(p<.01)$ this being due to linear trend which was significant $(p<.01)$, no other effects even approached significance; (d) $T_{5}$ vs $T_{6}$ (assessing effects of shift from DAF to VDAF, and vice versa, at end)-the levels effect was again significant $(p<.01)$, and so was that attributable to trials $(p<.05)$ indicating some improvement from $T_{5}$ to $\mathrm{T}_{6}$ even with a switch in conditions of delay in feedback.

In each case the levels effect which was significant reflected the fact that reading time under conditions of delayed feedback was positively correlated with reading time under normal conditions. The anticipation that this might be the case was, of course, the reason for using normal reading time as a control variable. These findings with regard to the levels effect are themselves of no particular interest.

The general tenor of the results is quite clear: (a) with regard to level of impairment there is no significant difference between DAF .20 and VDAF, (b) in both conditions significant improvement or adaptation does occur, the adaptation effect being linear in this situation, and there is no difference between conditions in rate or degree of adaptation (the between group trends effects are very small and nowhere near significance). In short, the two conditions do not differ, and adaptation does occur. ${ }^{3}$

The results do not bear out the analogy, mentioned earlier, between VDAF and a situation of variable visual dis-arrangement (brought about by employing prisms of continuously changing power). It is hard

to say why the results are so different with regard to adaptation. However, one obvious difference between the two situations might be noted. The visual situation involves simultaneous or concurrent sources of information which do not match, whereas the VDAF situation involves varying serial or temporal discrepancies in information.

What are the implications of the above results for the general interpretation of the effects of delay in auditory feedback? The data obtained in this study argue against any simple version of a mis-match hypothesis which seeks to interpret the effects of DAF in terms of asynchrony between different sources of feedback. For if asynchrony in feedback were the principal determinant of DAF effects then under conditions of VDAF one should perhaps expect even greater impairment. Or at the very least one should expect slower adaptation to the delay in feedback since the asynchrony is not constant, for it should be more difficult to come to cope with a variable, unpredictable disarrangement or mis-match than with a constant predictable one.

\section{References}

Teuber, H.-L. Perception. In J. Field, H. W. Magoun, and V. E. Hall (Eds.), Handbook of physiology, Vol. 3. Washington, D. C.: American Physiological Society, 1960.

Yates, A. J. Delayed auditory feedback. Psychol. Bull., 1963, 60, 213-232.

\section{Notes}

1. This research was supported by PHS Research Grant M-1849 from the National Institute of Mental Health, Public Health Service. The paper was completed while the author was a Research Fellow at Harvard University, Center for Cognitive Studies. The author is greatly indebted to Nancy Stooksberry for running the Ss and analyzing the data.

2. In fact, more Ss were run but some were randomly eliminated so as to have an equal number of Ss at each level in each condition.

3 . Analyses using change scores (defined in terms of the difference between reading time under the normal condition and conditions of delay in feedback) yielded exactly the same results. 